\title{
Effects of prebiotic inulin-type fructans on blood metabolite and hormone concentrations and faecal microbiota and metabolites in overweight dogs
}

\author{
Celeste Alexander ${ }^{1}$, Tzu-Wen L. Cross ${ }^{1}$, Saravanan Devendran ${ }^{2}$, Franka Neumer ${ }^{3}$, Stephan Theis $^{3}$, \\ Jason M. Ridlon ${ }^{1,2}$, Jan S. Suchodolski ${ }^{4}$, Maria R. C. de Godoy ${ }^{1,2}$ and Kelly S. Swanson ${ }^{1,2 *}$ \\ ${ }^{1}$ Division of Nutritional Sciences, University of Illinois at Urbana-Champaign, $1207 \mathrm{~W}$. Gregory Drive, Urbana, IL 61801, USA \\ ${ }^{2}$ Department of Animal Sciences, University of Illinois at Urbana-Champaign, $1207 \mathrm{~W}$. Gregory Drive, Urbana, IL 61801, USA \\ ${ }^{3}$ BENEO-Institute, c/o BENEO GmbH, Wormser Str. 11, 67283 Obrigheim, Germany \\ ${ }^{4}$ Gastrointestinal Laboratory, Texas AEM University, 4474 TAMU, College Station, TX 77843, USA
}

(Submitted 4 January 2018 - Final revision received 13 June 2018 - Accepted 15 June 2018 - First published online 1 August 2018)

\section{Abstract}

Because obesity is associated with many co-morbidities, including diabetes mellitus, this study evaluated the second-meal effect of a commercial prebiotic, inulin-type fructans, and the effects of the prebiotic on faecal microbiota, metabolites and bile acids (BA). Nine overweight beagles were used in a replicated $3 \times 3$ Latin square design to test a non-prebiotic control (cellulose) against a low (equivalent to $0.5 \%$ diet) and high dose (equivalent to $1.0 \%$ diet) of prebiotic over 14-d treatments. All dogs were fed the same diet twice daily, with treatments provided orally via gelatin capsules before meals. On days 13 or 14 of each period, fresh faecal samples were collected, dogs were fed at 08.00 hours and then challenged with $1 \mathrm{~g} / \mathrm{kg}$ body weight of maltodextrin in place of the 16.00 hours meal. Repeated blood samples were analysed for glucose and hormone concentrations to determine postprandial incremental AUC (IAUC) data. Baseline glucose, insulin and active glucagon-like peptide-1 levels were similar between all groups $(P>0 \cdot 10)$. Glucose and insulin IAUC after glucose challenge appeared lower following the high dose, but did not reach statistical relevance. Prebiotic intervention resulted in an increase in relative abundance of some Firmicutes and a decrease in the relative abundance of some Proteobacteria. Individual and total faecal SCFA were significantly increased $(P<0 \cdot 05)$ following prebiotic supplementation. Total concentration of excreted faecal BA tended to increase in dogs fed the prebiotic $(P=0 \cdot 06)$. Our results indicate that higher doses of inulin-type prebiotics may serve as modulators of gut microbiota, metabolites and BA pool in overweight dogs.

Key words: Bile acids: Canine nutrition: Second-meal effect: SCFA

Obesity is one of the most common diseases present in both humans and companion animals, and is often associated with many co-morbidities, including diabetes mellitus. Similar to obese humans, obese dogs often exhibit altered blood glucose concentrations, insulin resistance and dyslipidaemia ${ }^{(1-3)}$. Many human and canine studies have demonstrated the ability of dietary fibres, especially soluble fibres and prebiotics, to decrease postprandial hyperglycaemia ${ }^{(4,5)}$, improve insulin sensitivity ${ }^{(6,7)}$ and increase secretion of intestinal glucagon-like peptide-1 (GLP-1 $)^{(4,8)}$. Thus, fibres and prebiotics may be used to improve the postprandial glycaemic response in obese or overweight dogs. However, the efficacy of fibre and other nondigestible carbohydrates in producing such effects is largely dependent on source. The physiochemical properties of these ingredients, such as fermentability, may be quite different and lead to differing effects in vivo, stressing the importance of understanding which ingredients elicit a beneficial response ${ }^{(9)}$. For example, healthy dogs fed a high-fermentable fibre diet (beet pulp, gum arabic, fructo-oligosaccharides (FOS)) compared with those fed a low-fermentable fibre diet (wood cellulose) exhibited a blunted blood glucose response and an increased production of insulin and GLP-1 following an oral glucose challenge ${ }^{(4)}$. In addition, highly fermentable short-chain FOS supplementation in obese dogs has been shown to improve insulin sensitivity and modulate the expression of genes involved in fatty acid and glucose metabolism in $\operatorname{adipocytes}^{(6)}$

Interestingly, some studies have suggested that certain fibres and prebiotics can beneficially alter the glycaemic response many hours after a meal has been consumed. Such a response has been called the 'second-meal effect', and was first reported in humans in the early twentieth century ${ }^{(10,11)}$. A previous study conducted in our laboratory demonstrated that when dogs were fed a high-fermentable fibre diet (5\% pectin; 3\% inulin-type fructans) compared with a low-fermentable fibre diet ( $5 \%$ Solka Floc; 3\% soya hulls), they exhibited a decreased postprandial 
blood glucose response at a subsequent meal $4 \mathrm{~h}$ after consumption $^{(12)}$. Given that obese dogs frequently exhibit an impaired glycaemic response, including hyperglycaemia, hyperinsulinaemia and insulin resistance, the consumption of fermentable fibres may improve these measures across multiple meals. In addition, human studies have shown that consumption of initial meals high in fibre, especially those that are soluble and/or fermentable, can curb the glycaemic response of a high-glycaemic second meal ${ }^{(13-16)}$. The overarching hypothesis is that the production of SCFA (acetate; butyrate; propionate) produced from fermentation of fibre in the large bowel modulates the observed effects by delaying gastric emptying $^{(17)}$, down-regulating lipolysis in adipocytes ${ }^{(18)}$, increasing glucose utilisation in glycolysis and decreasing glucose production $^{(19)}$ and increasing postprandial insulin and GLP-1 production $^{(8)}$. Inulins are of particular interest given their high degree of fermentability by gut bacteria to produce SCFA. In addition to fibre type, it is important to determine dosages that are both adequate to produce a beneficial response and feasible for incorporation into the diet. To our knowledge, there has not been previous research investigating the effects of practical doses of inulin on the second-meal effect in overweight dogs.

It is well established that (1) body composition (lean $v$. obese) and (2) dietary fibres/prebiotics affect the composition and diversity of the gastrointestinal microbiome ${ }^{(20-25)}$. These effects suggest that prebiotics may be a promising dietary strategy for promoting health, especially in overweight or obese populations. However, little research has been performed in dogs using modern high-throughput DNAsequencing-based assays. Therefore, an important next step is to characterise how particular dietary fibres shift the gastrointestinal microbiome in both lean and overweight dogs. The study conducted here is among the first to investigate the impact of inulin-type prebiotics on the gut microbiota composition and diversity of overweight dogs. The gastrointestinal microbiota is also affected by bile acids (BA), and vice versa. Although BA possess antimicrobial properties by acting as detergents on microbial cell membranes, some bacteria in the gut possess enzymes that modify BA, rendering them less harmful to microbes. Because BA possess endocrine functionality, altering the composition of the BA pool may influence host metabolism ${ }^{(26,27)}$. The complex relationship that exists between dietary fibre, the gut microbiome and BA metabolism remains to be elucidated, especially in dogs. To our knowledge, we are the first to determine the effect of an inulin product on the faecal BA pool in dogs.

Therefore, the objectives of this study were (1) to evaluate the effects of inulin on the baseline and postprandial glucose, insulin and active GLP-1 responses to an oral glucose challenge in overweight dogs several hours after its consumption, and (2) to evaluate the effects of inulin on the faecal microbiota and BA pool in overweight dogs. On the basis of previous studies, we hypothesised that the prebiotic would elicit a 'second-meal effect' and beneficially shift the faecal microbiota, resulting in a greater relative abundance of Bifidobacterium, Faecalibacterium, Akkermansia and Lachnospira and lower relative abundance of Fusobacterium and Desulfovibrio.

\section{Methods}

\section{Animals, diet and treatments}

All procedures were approved by the University of Illinois Institutional Animal Care and Use Committee before the study (protocol no. 15248). Nine overweight adult (mean age $=4 \cdot 16$ (sEM 0.67) years) female beagles (mean body weight $(\mathrm{BW})=$ $12.96($ SEM 2.42$) \mathrm{kg}$; mean body condition score $(\mathrm{BCS})=7 \cdot 8(\mathrm{sEM}$ $1.4)$ ) were used in this study and were housed individually in pens $(1.2 \mathrm{~m}$ wide $\times 1.8 \mathrm{~m}$ long) in a temperature-controlled room $\left(20^{\circ} \mathrm{C}\right)$ on a $14 \mathrm{~h}$ light $-10 \mathrm{~h}$ dark cycle in the animal facility at the Veterinary Medicine Basic Sciences Building at the University of Illinois. An overweight phenotype was induced by overfeeding and maintained for several months before the beginning of the current study. Dogs were weighed and BCS were assessed using a nine-point scale once a week in the morning before feeding. Dogs were given access to toys for behavioural enrichment at all times and to exercise outside of their cages and socialise with each other and humans for approximately $1 \mathrm{~h}$ at least $3 \mathrm{~d} /$ week. Dogs were evaluated and shown to be healthy before the study and after study completion. Welfare was monitored by research and animal care staff daily and trained veterinarians during the study when needed.

The ingredient and chemical composition of the experimental diet is listed in Table 1 . The diet was mixed by Lortscher Animal Nutrition, manufactured by AFB International, and formulated to meet all Association of American Feed Control Officials (2015) nutrient recommendations for adult dogs. All

Table 1. Ingredient and analysed chemical composition of the experimental diet

\begin{tabular}{|c|c|}
\hline & $\%$ As-fed \\
\hline \multicolumn{2}{|l|}{ Ingredients } \\
\hline Chicken by-product meal (low-ash) & 49.00 \\
\hline Brewer's rice (US no. 4) & 33.36 \\
\hline Chicken fat & $9 \cdot 00$ \\
\hline Maize whole (US no. 2) & 5.00 \\
\hline Cellulose - Solka Floc 900 & 2.00 \\
\hline Salt (sodium chloride) & 0.50 \\
\hline Potassium chloride $(50 \% \mathrm{~K})$ & 0.45 \\
\hline Taurine & 0.20 \\
\hline Mineral premix ${ }^{*}$ & 0.18 \\
\hline Vitamin premix $\dagger$ & 0.18 \\
\hline Choline chloride & 0.13 \\
\hline Analysed chemical composition & $\%$ \\
\hline DM & $91 \cdot 1$ \\
\hline & $\%$ DM basis \\
\hline Organic matter & 93.0 \\
\hline Crude protein & 38.6 \\
\hline Acid-hydrolysed fat & $17 \cdot 3$ \\
\hline Total dietary fibre & $7 \cdot 2$ \\
\hline Gross energy (kJ/g DM basis) & $22 \cdot 18$ \\
\hline Calculated ME (kJ/g DM basis) & $16 \cdot 19$ \\
\hline \multicolumn{2}{|c|}{ 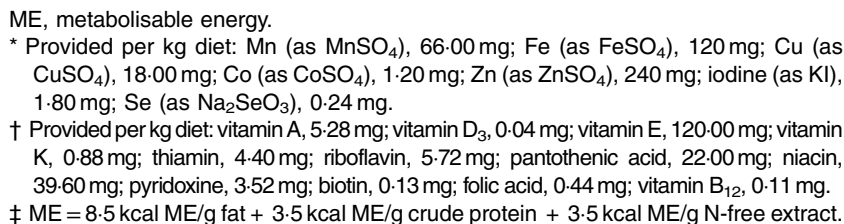 } \\
\hline
\end{tabular}


dogs were fed the same experimental diet for the duration of the study. Initial food intake was determined by calculating the maintenance energy requirement according to the National Research Council ${ }^{(28)}$ and by using previous feeding records. Dogs had access to fresh water ad libitum and were fed twice a day (08.00 and 16.00 hours) to maintain BW throughout the study. Food intake and refusals were measured and recorded at each meal. Prebiotic treatments (Orafti ${ }^{\circledR}$ SIPX; Beneo GmbH) were given to dogs via gelatin capsules at each meal, with capsules containing half the daily dose. The total dose of each treatment/d was as follows: (1) placebo (cellulose, equivalent to approximately $0.25 \%$ of diet, $0.5 \mathrm{~g} / \mathrm{d}$ ); (2) Orafti ${ }^{\circledR} \mathrm{SIPX}$ (equivalent to approximately $0.5 \%$ of diet, $1 \mathrm{~g} / \mathrm{d}$ ); and (3) Orafti ${ }^{\circledR}$ SIPX (equivalent to approximately $1 \%$ of diet, $2 \mathrm{~g} / \mathrm{d}$ ). Dosage was selected based on previous work in our laboratory investigating prebiotic fructans, including impacts on the microbiome and benefits to host health, as well as on inclusion feasibility from a consumer cost perspective $\mathrm{e}^{(20,29,30)}$.

\section{Experimental design}

Dogs were randomised to an initial treatment group (control, low dose or high dose), and a replicated $3 \times 3$ Latin square design was used. Using this design, each dog received all three treatments over the course of the experiment, serving as their own control and increasing statistical power ( $n$ 9). The experiment consisted of three 14-d experimental periods separated by 14-d washout periods. Each experimental period consisted of an adaption phase (days 1-12) and a faecal and blood collection phase (days 13-14).

\section{Blood and faecal sample collection}

On days 13 and 14, a fresh (within 15 min of defecation) faecal sample was collected from the pen floor of each dog. All samples were scored in 0.5 unit increments using the following scale: $1=$ hard, dry pellets; small hard masses, $2=$ hard, formed, dry stool; remains firm and soft, $3=$ soft, formed, and moist stool; retains shape, $4=$ soft, unformed stool; assumes shape of container, $5=$ watery, liquid that can be poured. Faecal $\mathrm{pH}$ was measured immediately, and then aliquots were collected. Aliquots for phenol and indole measurement were frozen and stored at $-20^{\circ} \mathrm{C}$ until analysis. An aliquot for ammonia, SCFA and branched-chain fatty acid (BCFA) measurement was placed in $2 \mathrm{M}-\mathrm{HCl}$ and stored at $-20^{\circ} \mathrm{C}$ until analysis. An aliquot was collected for the determination of faecal DM determination. Finally, aliquots for microbiota and BA measurement were transferred to sterile cryogenic vials (Nalgene), frozen on dry ice and stored at $-80^{\circ} \mathrm{C}$ until analysis.

On days 13 and 14, an oral glucose tolerance test (OGTT) was also performed. On those days, dogs were fed at 08.00 hours as usual. Dogs were sedated using dexmedetomadine (Dexdomitor, $0.02 \mathrm{mg} / \mathrm{kg} \mathrm{BW}$ intramuscular (IM); Orion Pharma) and butorphanol (Torbugesic, $0.2 \mathrm{mg} / \mathrm{kg} \mathrm{BW} \mathrm{IM,}$ Henry Schein) at 14.00 hours for approximately 15 min during cephalic catheter placement. Once the catheters were placed and secure, dogs were given atipamezole (Antisedan, $0.02 \mathrm{mg} / \mathrm{kg}$ BW IM; Orion Pharma), the reversal agent for Dexdomitor, and monitored until awake and alert. Just before 16.00 hours, a blood sample was collected for baseline glucose, insulin and GLP-1 measurement. Instead of their 16.00 hours meal, dogs received an oral glucose load ( $1 \mathrm{~g} / \mathrm{kg} \mathrm{BW})$ of maltodextrin as a $50 \%$ solution by slowly dripping it into their mouth using $60-\mathrm{ml}$ needleless syringes to minimise stress and prevent aspiration. After dosing, blood samples were collected after 10, 20, 30, 45, 60, 90, 120 and $180 \mathrm{~min}$ from the cephalic catheters. After $180 \mathrm{~min}$, catheters were removed and dogs were given their evening meal (19.00 hours).

\section{Chemical analysis of diet}

Diet samples were ground using a Wiley mill (model 4; Thomas Scientific) through a 2-mm screen and analysed for DM, organic matter and ash according to the Association of Official Analytical Chemists ${ }^{(31)}$. Crude protein was calculated from Leco (FP2000 and TruMac) total N values according to Association of Official Analytical Chemists ${ }^{(31)}$. Total lipid content (acidhydrolysed fat) was determined according to the methods of the American Association of Cereal Chemists ${ }^{(32)}$ and Budde ${ }^{(33)}$. Total dietary fibre was determined according to Prosky et al. ${ }^{(34)}$. Gross energy was measured using an oxygen bomb calorimeter (model 1261; Parr Instruments).

\section{Serum and plasma analysis}

One drop of blood from the syringe was immediately used to measure glucose concentration using the glucose oxidase method (AlphaTRAK Blood Glucose Monitoring System; Abbott Laboratories) at each time point. An aliquot was immediately transferred into a pre-cooled Vacutainer tube (no. 367835; Becton, Dickinson and Company) containing EDTA and $20 \mu \mathrm{l}$ of dipeptidyl peptidase IV inhibitor $(10 \mu \mathrm{l} / \mathrm{ml}$ blood; Millipore), and centrifuged (GS-6R; Beckman) at $1000 \mathrm{~g}$ at $4^{\circ} \mathrm{C}$ for $10 \mathrm{~min}$ for measurement of plasma active GLP-1. The remaining blood was transferred to a serum separator tube (no. 367985; Becton, Dickinson and Company), allowed to clot and centrifuged at $1300 \mathrm{~g}$ at room temperature for $15 \mathrm{~min}$ for collection of serum for insulin measurement. After centrifugation, supernatants were collected and stored into respective cryovials and stored at $-20^{\circ} \mathrm{C}$ (serum) or $-80^{\circ} \mathrm{C}$ (plasma) until further analysis. Serum insulin concentration was determined using a Rat Insulin EIA kit (Bertin Pharma) validated for use in dogs ${ }^{(35,36)}$. Standard curves were generated using the Gen 5 software and a 4 Parameter fit. Plasma active GLP-1 concentrations were analysed using a Glucagon-Like Peptide 1 (Active) ELISA kit (Millipore) validated for use in $\operatorname{dogs}{ }^{(36)}$

\section{Faecal metabolite analysis}

Faecal SCFA and BCFA concentrations were determined using GC according to Erwin et al. using a gas chromatograph (Hewlett-Packard 5890A series II; Agilent Technologies) and a glass column $(180 \mathrm{~cm} \times 4 \mathrm{~mm}$ internal diameter $)$ packed with $10 \%$ SP-1200/1 \% H3PO4 on 80/100 + mesh Chromosorb WAW (Supelco Inc.) ${ }^{(37)} \cdot \mathrm{N}_{2}$ was the carrier with a flow rate of $75 \mathrm{ml} /$ min. Oven, detector and injector temperatures were 125, 175 
and $180^{\circ} \mathrm{C}$, respectively. Faecal ammonia concentration was determined according to the method of Chaney \& Marbach ${ }^{(38)}$. Faecal phenol and indole concentrations were determined using GC according to the methods described by Flickinger et $a l .{ }^{(39)}$.

For faecal BA analysis, stool samples were lyophilised and BA extraction was performed using Method B (alkaline hydrolysis) according to Kakiyama et al. without any modifications $^{(40)}$. For BA analysis, HPLC equipment (Shimadzu) equipped with a C-18 analytical column (Capcell Pak c18; Shiseido) was used. Methanol (82\%) was used as the mobile phase and the flow rate was maintained at $0.2 \mathrm{ml} / \mathrm{min}$. A diode array detector was used at a wavelength of $254 \mathrm{~nm}$. Peak retention times and peak areas of samples were compared with internal standard (IS) steroid molecules (Steraloids). Calculations of BA concentrations were performed using the following equation:

$$
\mathrm{BA}(\mu \mathrm{mol} / \mathrm{g} \mathrm{stool})=\frac{\text { Peak area }(\mathrm{BA})}{\text { Peak area }(\mathrm{IS})} \times \frac{\text { Spiked IS }(\mathrm{nmol})}{\text { Stool amount }(\mathrm{mg})} .
$$

\section{Faecal microbiota}

Faecal bacterial DNA was extracted according to McInnes \& Cutting $^{(41)}$ using the MO BIO PowerLyzer PowerSoil Kit (MO BIO Laboratories) with bead beating using a vortex adaptor. The concentration of extracted DNA was quantified using a Qubit $^{\circledR}$ 3.0 Fluorometer (Life Technologies). 16S rRNA gene amplicons were generated using a Fluidigm Access Array (Fluidigm Corporation) in combination with Roche High Fidelity Fast Start Kit (Roche). The primers 515F (5'-GTGYCAGCMG CCGCGGTAA-3') and 806R (5'-GGACTACNVGGGTWTCT AAT-3') that target a 252-bp fragment of the V4 region were used for amplification (primers synthesised by IDT Corp. ${ }^{(42)}$. CS1 forward tag and CS2 reverse tag were added according to the Fluidigm protocol. Quality of the amplicons was assessed using a Fragment Analyzer (Advanced Analytics) to confirm amplicon regions and sizes. A DNA pool was generated by combining equimolar amounts of the amplicons from each sample. The pooled samples were then size-selected on a $2 \%$ agarose E-gel (Life technologies) and extracted using a Qiagen gel purification kit (Qiagen). Cleaned size-selected pooled products were run on an Agilent 2100 Bioanalyzer to confirm appropriate profile and average size. Sequencing was performed at the W. M. Keck Center for Biotechnology at the University of Illinois using an Illumina MiSeq using version 3 reagents (Illumina Inc.).

Quantitative PCR was performed according to previous methods ${ }^{(43,44)}$. Briefly, primers targeting all bacteria (universal primer), specific genera (Lactobacillus, Bifidobacterium, Blautia, Faecalibacterium and Fusobacterium) and specific species (Escherichia coli and Clostridium perfringens) were used. Real-time PCR conditions and primers used for Lactobacillus spp., Fusobacterium spp., Blautia spp., Faecalibacterium spp., Bifidobacterium spp. and C. perfringens have been described by Rossi et al. ${ }^{(44)}$. Real-time PCR conditions and primers for $E$. coli have been described by Malinen et al. ${ }^{(43)}$. Briefly, standard curves were generated using DNA (ranging from $2 \mathrm{ng}$ to $0.2 \mathrm{pg}$ ) from lyophilised bacterial species of the genera (Faecalibacterium prausnitzii (ATCC 27766); Lactobacillus rhamnosus GG (ATCC 53103); Bifidobacterium bifidum (ATCC 11863); Blautia coccoides (ATCC 29236); Fusobacterium nucleatum (ATCC 25586)), clinical isolates from E. coli and C. perfringens and canine faecal community DNA for universal bacteria. The quantitative PCR (qPCR) data were expressed as log amount of DNA (fg) for each particular bacterial group per $10 \mathrm{ng}$ of isolated total $\mathrm{DNA}^{(45)}$.

High-quality (quality value $>19$ ) sequence data derived from the sequencing process were analysed using QIIME 1.9.1 ${ }^{(46)}$. Briefly, sequence data derived from the sequencing process were demultiplexed. Sequences then were clustered into operational taxonomic units (OTU) using UCLUST ${ }^{(47)}$ through a closed-reference OTU picking strategy against the Greengenes 13 _8 reference database ${ }^{(48)}$ with a $97 \%$ similarity threshold. Singletons (OTU that were observed fewer than two times) and OTU that had less than $0.01 \%$ of the total observation were discarded. Taxonomic identity to each OTU was then assigned using UCLUST. A total of 2345512 16S rRNA-based amplicon sequences were obtained, with an average of 86870 reads (range $=26961-368990$ ) per sample. An even sampling depth (sequences per sample) of 26961 sequences per sample was used for assessing alpha- and beta-diversity measures. Betadiversity was calculated using weighted and unweighted UniFrac distance measures ${ }^{(49)}$.

\section{Statistical analysis}

On the basis of our previous feline (Deng et al. ${ }^{(12)} ; n$ 12) and canine (Lubbs et al. ${ }^{(36)}, n$ 8; Deng et al. ${ }^{(50)}, n$ 6) experiments in this area, we estimated the need for nine dogs per treatment to reach a significance level of 0.05 with a power of $80 \%$ for the variables of interest ${ }^{(12,36,50)}$. As in our previous studies, we used a replicated $3 \times 3$ Latin square design so that each animal received each treatment/control provided. This design allowed the animals to serve as a self-control, reducing outcome variation and the number of animals needed.

All data, except faecal microbiota data, were analysed using SAS (version 9.3; SAS Institute) using the Mixed Models procedure, testing the main effect of experimental diet and including random effect of dog. Incremental AUC (IAUC) $0-180$ min data for glucose, active GLP-1 and insulin concentrations were calculated using all positive peak areas using GraphPad Prism version 6.0 (GraphPad Software) and statistically analysed using the Mixed Models procedure as repeated measures using 'dog' as a random variable. Data are reported as least square means with their standard errors with statistical significance set as $P<0.05$ and $P<0.10$ considered as trends. Faecal microbiota data were analysed using ANOVA and Tukey-Kramer multiple comparison tests with Statistical Analysis of Metagenomic Profiles software 2.1.3 ${ }^{(51)}$. Microbiota data are reported as means and standard deviations with statistical significance set as $P<0.05$ and $P<0.10$ considered as trends.

\section{Results}

No adverse events were observed following prebiotic supplementation in any of the treatment groups. Food intake was rather comparable between dogs fed the high dose of prebiotic $(175.4 \mathrm{~g} / \mathrm{d})$ and those of the control group $(168.1 \mathrm{~g} / \mathrm{d})$, but it 
Table 2. Blood hormone and metabolite concentrations of prebiotic-supplemented dogs in response to an oral glucose tolerance test several hours after consumption of different prebiotic doses (Mean values with their standard errors)

\begin{tabular}{|c|c|c|c|c|c|}
\hline & \multicolumn{3}{|c|}{ Treatment } & \multirow[b]{2}{*}{ Pooled SEM } & \multirow[b]{2}{*}{$P$} \\
\hline & Control & Low & High & & \\
\hline \multicolumn{6}{|l|}{ Baseline concentration } \\
\hline Glucose $(\mathrm{mg} / \mathrm{dl})^{\star}$ & 91.53 & 85.82 & $89 \cdot 44$ & $5 \cdot 71$ & 0.70 \\
\hline Insulin (pmol/l) & $30 \cdot 86$ & $24 \cdot 60$ & $32 \cdot 32$ & 8.09 & 0.61 \\
\hline Active GLP-1 (pmol/l) & 6.79 & 7.49 & $6 \cdot 31$ & 1.42 & 0.84 \\
\hline Insulin:glucose† & 0.36 & 0.31 & 0.40 & 0.11 & 0.77 \\
\hline \multicolumn{6}{|l|}{$I^{A U C} C_{0-180 \mathrm{~min}}$} \\
\hline Glucose (mg/dl)* & $2453 \cdot 1$ & 3734.8 & $1629 \cdot 4$ & $841 \cdot 1$ & 0.29 \\
\hline Insulin (pmol/l) & $6858 \cdot 4$ & 6893.8 & 4594.2 & $1569 \cdot 8$ & 0.46 \\
\hline Active GLP-1 (pmol/l) & $800 \cdot 4$ & $908 \cdot 2$ & $583 \cdot 1$ & $326 \cdot 9$ & 0.24 \\
\hline Insulin:glucose $\mid \mathrm{AUC}_{0-180 \mathrm{~min}}$ & 3.61 & $1 \cdot 87$ & 2.87 & 1.06 & 0.57 \\
\hline
\end{tabular}

IAUC, incremental AUC.

* To convert glucose from $\mathrm{mg} / \mathrm{dl}$ to $\mathrm{mmol} / \mathrm{l}$, multiply by 0.0555 .

† Fasting baseline insulin:glucose ratio.
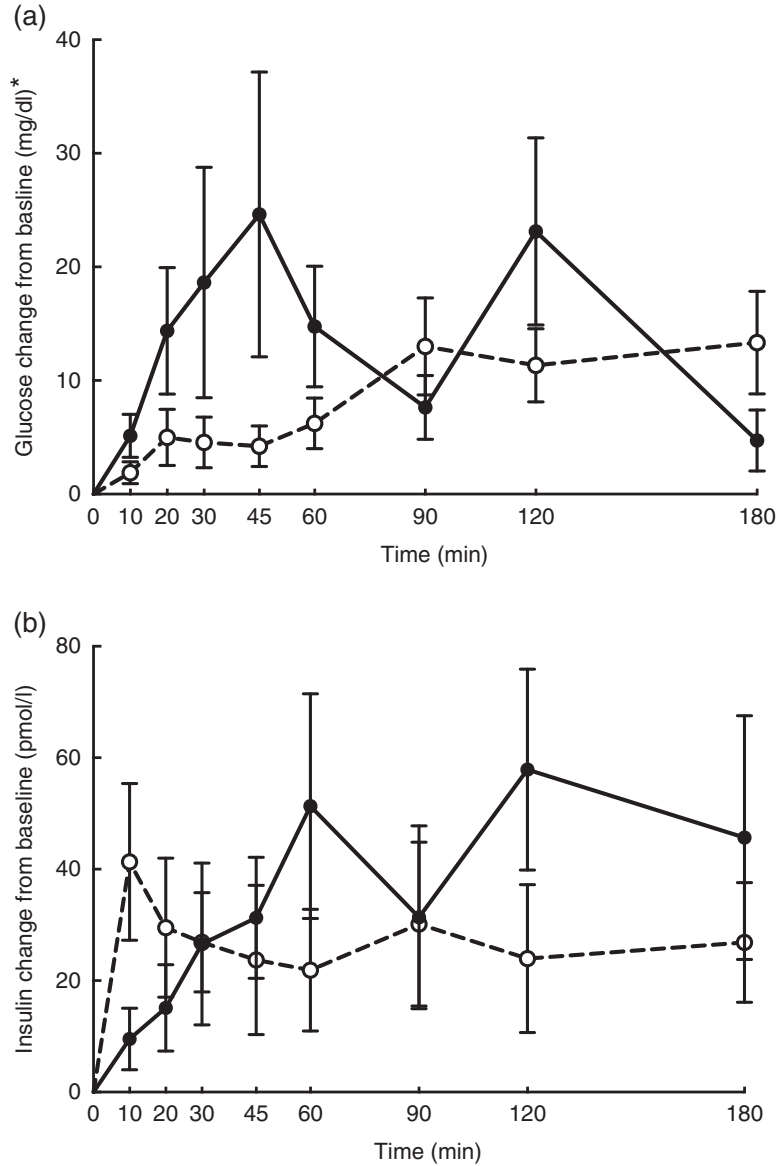

Fig. 1. Glucose (a) and insulin (b) concentrations in response to an oral glucose challenge test in dogs $8 \mathrm{~h}$ after consumption of $1 \%$ of Orafti ${ }^{\circledR} \mathrm{SIPX}$ (high-dose prebiotic; $\bigcirc,----) v$. control $\left(O_{-}-\right)$). * To convert glucose from $\mathrm{mg} / \mathrm{dl}$ to $\mathrm{mmol} / \mathrm{l}$, multiply by 0.0555 .

was greater $(P<0.05)$ compared with dogs given the low-dose prebiotic $(160.7 \mathrm{~g} / \mathrm{d})$. There were no differences among prebiotic treatments for baseline glucose, insulin or active GLP-1 concentrations. The respective postprandial $\mathrm{IAUC}_{0-180}$ values were numerically the lowest for dogs fed the high prebiotic dose, but did not reach statistical significance (Table 2, Fig. 1).
Faecal characteristics and metabolites were affected by treatment (Table 3). Faecal DM percentage was lower $(P<0.05)$ in dogs fed the high prebiotic dose compared with dogs fed the control, with dogs fed the low prebiotic dose being intermediate. Faecal $\mathrm{pH}$ and scores were not different among treatment groups. Faecal acetate concentrations were higher $(P<0.05)$ in dogs fed the high prebiotic dose compared with those fed the low prebiotic dose or control. Faecal butyrate and propionate concentrations were higher $(P<0.05)$ in dogs fed the high prebiotic dose than those fed the low prebiotic dose, with controls being intermediate. Total SCFA concentrations were higher $(P<0.05)$ in dogs fed the high prebiotic dose compared with those fed either the low prebiotic dose or control. There was a trend for greater $(P<0 \cdot 10)$ faecal valerate concentrations in dogs fed the high prebiotic dose compared with dogs fed the control, with the dogs fed low prebiotic dose being intermediate. Faecal isovalerate, isobutryate, total BCFA, phenol, indole, total phenol + indole and ammonia concentrations were not significantly affected by treatment. Faecal deoxycholic acid (DCA), lithocholic acid (LCA) and total BA concentrations tended to be affected by treatment $(P<0 \cdot 10)$, with dogs fed the high prebiotic dose having numerically greater values than dogs fed the low prebiotic dose.

Faecal microbial species richness (alpha diversity; online Supplementary Fig. S1) was not affected by treatment. Neither unweighted nor weighted principal coordinate analysis plots of UniFrac distances (beta diversity) of faecal microbial communities (online Supplementary Fig. S2) revealed significant differences among treatments. Regarding specific microbial taxa, there were a few changes in the Erysipelotrichaceae family (Table 4). Faecal Coprobacillus was lower $(P<0.05)$ in dogs fed the high prebiotic dose compared with those fed the control, with dogs fed the low prebiotic dose being intermediate. Faecal Eubacterium tended to be greater $(P<0 \cdot 10)$ in dogs fed the high prebiotic dose compared with dogs fed the control, with dogs fed the low prebiotic dose being intermediate. Faecal Erysipelotrichaceae, Turicibacter and Anaerobiospirillum tended to be different among treatments. Of the faecal microbiota measured by qPCR, Turicibacter was higher $(P<0.05)$ in dogs fed the high prebiotic dose than those fed the control or low prebiotic dose (online Supplementary Table S1). None of the other microbiota were 
Table 3. Fresh faecal characteristics of prebiotic-supplemented dogs

(Mean values with their standard errors)

\begin{tabular}{|c|c|c|c|c|c|}
\hline & \multicolumn{3}{|c|}{ Treatment } & \multirow[b]{2}{*}{ SEM } & \multirow[b]{2}{*}{$P$} \\
\hline & Control & Low & High & & \\
\hline \multicolumn{6}{|l|}{ Faecal characteristics } \\
\hline DM (\%) & $40 \cdot 36^{a}$ & $39.37^{\mathrm{a}, \mathrm{b}}$ & $37.44^{\mathrm{b}}$ & 1.02 & 0.04 \\
\hline $\mathrm{pH}$ & 6.57 & 6.68 & 6.54 & 0.12 & 0.67 \\
\hline Faecal score* & 2.56 & $2 \cdot 50$ & $2 \cdot 50$ & 0.15 & 0.93 \\
\hline \multicolumn{6}{|l|}{ Faecal metabolites ( $\mu \mathrm{mol} / \mathrm{g}$ DM basis) } \\
\hline Acetate & $180 \cdot 24^{\mathrm{a}}$ & $173 \cdot 21^{\mathrm{a}}$ & $230.94^{\mathrm{b}}$ & 13.68 & $<0.01$ \\
\hline Butyrate & $38 \cdot 67^{\mathrm{a}, \mathrm{b}}$ & $34.03^{\mathrm{a}}$ & $47.79^{\mathrm{b}}$ & 3.72 & 0.02 \\
\hline Propionate & $102 \cdot 37^{a, b}$ & $96 \cdot 52^{\mathrm{a}}$ & $130 \cdot 13^{\mathrm{b}}$ & 10.91 & 0.02 \\
\hline Total SCFA† & $321 \cdot 28^{a}$ & $303.75^{a}$ & $408 \cdot 86^{b}$ & $25 \cdot 38$ & $<0.01$ \\
\hline Isobutyrate & 9.37 & 9.33 & 10.47 & 0.99 & 0.46 \\
\hline Isovalerate & $13 \cdot 36$ & 12.44 & 13.50 & 1.36 & 0.71 \\
\hline Valerate & $0.77^{\times}$ & $0.82^{x, y}$ & $1 \cdot 15^{\mathrm{y}}$ & 0.11 & 0.05 \\
\hline Total BCFA $†$ & 23.50 & 22.59 & $25 \cdot 13$ & $2 \cdot 35$ & 0.58 \\
\hline Phenol & 0.82 & 0.98 & 0.86 & 0.31 & 0.65 \\
\hline Indole & 1.94 & $2 \cdot 10$ & 1.58 & 0.29 & 0.15 \\
\hline Total phenols + indoles & $2 \cdot 76$ & 3.08 & 2.43 & 0.37 & 0.16 \\
\hline Ammonia & $206 \cdot 16$ & $214 \cdot 31$ & $224 \cdot 30$ & 18.79 & 0.75 \\
\hline \multicolumn{6}{|l|}{ Bile acids } \\
\hline Cholic acid & 5.41 & 4.99 & 6.63 & 0.77 & 0.24 \\
\hline Deoxycholic acid & 49.87 & 41.80 & $60 \cdot 64$ & $5 \cdot 40$ & 0.08 \\
\hline Lithocholic acid & $15 \cdot 39$ & 11.30 & 16.09 & 1.89 & 0.09 \\
\hline 7-Oxo-deoxycholic acid & $25 \cdot 28$ & 24.23 & 28.05 & 3.42 & 0.52 \\
\hline 3-Oxo-chenodeoxycholic acid & 1.96 & 1.99 & $1 \cdot 78$ & 0.39 & 0.95 \\
\hline Iso-lithocholic acid & 4.06 & $4 \cdot 14$ & 4.25 & 0.50 & 0.95 \\
\hline Total bile acids & $102 \cdot 27$ & 88.45 & 117.45 & 7.74 & 0.06 \\
\hline
\end{tabular}

Table 4. Altered faecal microbiota populations of prebiotic-supplemented dogs using Illumina MiSeq sequencing

(Mean values and standard deviations)

\begin{tabular}{|c|c|c|c|c|c|c|c|c|c|}
\hline \multirow[b]{3}{*}{ Phylum } & \multirow[b]{3}{*}{ Family } & \multirow[b]{3}{*}{ Genus } & \multicolumn{6}{|c|}{ Treatment } & \multirow[b]{3}{*}{$P$} \\
\hline & & & \multicolumn{2}{|c|}{ Control } & \multicolumn{2}{|c|}{ Low } & \multicolumn{2}{|c|}{ High } & \\
\hline & & & Mean & SD & Mean & SD & Mean & SD & \\
\hline & & & & & $\%$ of $\mathrm{s}$ & nces & & & \\
\hline \multirow[t]{6}{*}{ Firmicutes } & & & 55.57 & $12 \cdot 51$ & $57 \cdot 15$ & $12 \cdot 83$ & $60 \cdot 38$ & $10 \cdot 70$ & NS \\
\hline & Erysipelotrichaceae & & 4.50 & 1.51 & 6.41 & 3.39 & 8.50 & 4.20 & 0.07 \\
\hline & & Coprobacillus & $0.13^{a}$ & 0.15 & $0.03^{a, b}$ & 0.03 & $0.01^{\mathrm{b}}$ & 0.01 & 0.02 \\
\hline & & (Eubacterium) & $0.65^{x}$ & 0.51 & $2 \cdot 65^{x, y}$ & $2 \cdot 34$ & $3.06^{y}$ & 2.37 & 0.05 \\
\hline & Turicibacteraceae & & 0.56 & 0.57 & 0.93 & 0.94 & 1.94 & 1.78 & 0.08 \\
\hline & & Turicibacter & 0.56 & 0.57 & 0.93 & 0.94 & 1.94 & 1.78 & 0.08 \\
\hline \multirow[t]{3}{*}{ Proteobacteria } & & & 3.75 & $2 \cdot 02$ & 3.92 & $2 \cdot 68$ & $2 \cdot 74$ & 1.06 & NS \\
\hline & Succinivibrionaceae & & 1.89 & 1.28 & $2 \cdot 38$ & 2.05 & 0.66 & 0.43 & 0.06 \\
\hline & & Anaerobiospirillum & 1.89 & $1 \cdot 28$ & $2 \cdot 38$ & 2.05 & 0.66 & 0.43 & 0.06 \\
\hline
\end{tabular}

a,b Mean values within a row with unlike superscript letters were different $(P<0.05)$.

${ }^{x, y}$ Mean values within a row with unlike superscript letters tended to be different $(P<0.10)$.

affected by treatment. There were no significant changes in the relative abundance of Bifidobacterium, Faecalibacterium, Lachnospira, Fusobacterium or Desulfovibrio as was hypothesised. Akkermansia was not detected in this study.

\section{Discussion}

Investigating the ability of various fibre sources and whole foods to produce a second-meal effect has gained considerable interest since Staub and Traugott first reported it in the early $1920 \mathrm{~s}^{(10,11)}$. Table 5 summarises and compares some of the more recent studies with the present study.

Previously, our laboratory demonstrated that fermentable fibre could produce a second-meal effect in healthy female dogs, reducing glucose IAUC $_{0-180 \text { min }}{ }^{(12)}$. In the present study, baseline glucose, insulin or GLP-1 concentrations were not different. The second-meal effect, based on IAUC for glucose, as well as the IAUC for insulin, and GLP-1, were not 
Table 5. Recent studies investigating the second-meal effect of fibres and prebiotics

\begin{tabular}{|c|c|c|c|c|}
\hline Subjects & Test product, dietary inclusion & Dosage time $(\mathrm{h})$ before OGTT & Evidence of second-meal effect & Reference \\
\hline Canine, overweight & Inulin, $0.5-1.0 \%$ & 8 & None & This study \\
\hline Canine, healthy & Pectin, $5 \%$; inulin-type fructans, $3 \%$ & 4 & $\downarrow$ Glucose IAUC & (12) \\
\hline Human, healthy & Lentils, $13.5 \mathrm{~g}$ fibre & 2 & $\downarrow$ Glucose IAUC & $(52)$ \\
\hline Human, type 2 diabetes & Psyllium fibre, $6.6 \mathrm{~g}$ & 4 & $\downarrow$ Insulin IAUC & (53) \\
\hline
\end{tabular}

OGTT, oral glucose tolerance test; IAUC, incremental AUC.

affected by treatment. However, the IAUC for glucose and insulin was numerically lower in dogs fed the high prebiotic dose.

In addition, there were a few important differences between this study and those previously conducted in this area. First, the fibre inclusion rate used in our previous study was much higher $(8 \%=5 \%$ pectin, $3 \%$ inulin-type fructans) than that supplemented in the current study (0.5\% or $1.0 \%$ of the diet). Second, the OGTT was conducted only $4 \mathrm{~h}$ after the morning meal in Deng et $a l^{(10)}$ rather than the $8 \mathrm{~h}$ used in the current study. It is important to note that all the other studies were designed to mimic human eating patterns (meals separated by $4-5 \mathrm{~h}$ ), whereas the present study was designed to mimic companion animal eating patterns in a typical household (meals separated by 8-9h). Last, overweight dogs were used in the current study rather than healthy adult dogs used in Deng et al. ${ }^{(10)}$ Therefore, when compared with the other studies, it could be suggested that the lack of effect in the current study was because of the lower inclusion of fermentable substrate in the diet $(1 \%=2 \mathrm{~g})$ or the extended amount of time between the morning meal and the OGTT.

In the current study, food intake was greater and faecal DM percentage was lower in dogs fed the high prebiotic dose. These were small numerical changes, however. Because all dogs consumed the diet without issues and maintained desirable faecal scores throughout the study, these differences are of little biological relevance. Faecal microbiota and metabolites were minimally altered owing to prebiotic treatment, but some beneficial shifts were noted. For example, faecal SCFA were greater in dogs fed the high prebiotic dose. The production of SCFA is beneficial to the host because they provide energy to colonocytes, reduce $\mathrm{pH}$ to limit pathogen growth and act as secondary messengers that stimulate gut peptide synthesis (i.e. GLP-1) and initiate other signal transduction pathways (i.e. increased glucose utilisation and decreased glucose production) ${ }^{(54-56)}$. The fermentation of protein produces some SCFA, but also a lot of putrefactive compounds including phenols, indoles, ammonia and biogenic amines that contribute to faecal odour and may be detrimental to colon health $^{(57,58)}$. Indole concentrations were numerically lower in dogs fed the higher prebiotic dose, but overall the protein fermentation products were not greatly altered by prebiotic supplementation in this study.

BA are efficiently reabsorbed (95\%) in the distal ileum. The $5 \%$ of BA that are not reabsorbed will reach the colon and be excreted in the faeces. While in the colon, some bacteria can convert primary BA (cholic acid (CA); chenodeoxycholic acid (CDCA)) into secondary BA (LCA; DCA) through dehydroxylation reactions ${ }^{(59)}$. Many additional organisms possess other BA metabolic activities, and thus changes to the microbiota can influence the BA pool ${ }^{(59,60)}$. Although there are very little data on faecal BA profiles in dogs, one small study $(n$ 3) characterised the BA present in the gallbladder and determined that $99 \%$ of them were CA, DCA or $\mathrm{CDCA}^{(61)}$. Another study showed that dogs with inflammatory bowel disease have a greater primary BA concentration and a lower secondary BA concentration compared with healthy controls ${ }^{(62)}$.

BA synthesis from endogenous cholesterol in the liver serves as the major route of cholesterol excretion from the body. Fibre supplementation has been shown to lower blood cholesterol concentrations in humans, probably because of increased BA excretion. Rémésy et $a l .{ }^{(63)}$ reported that inulin supplementation in mice made BA less soluble and enhanced their excretion in faeces. It is hypothesised that this is because of the production of SCFA from inulin fermentation, which lowers the luminal $\mathrm{pH}$ of the small intestine and hinders BA reabsorption. To our knowledge, we are the first to investigate changes in the faecal BA pool after inulin supplementation in dogs. In this study, faecal LCA, DCA and total BA tended to be affected by prebiotic supplementation. Our finding that inulin supplementation, at $1 \%$ of the diet, tended to increase total faecal BA supports this hypothesis.

It is well established that fermentable fibres and prebiotics can alter the gastrointestinal microbiota. Although we hypothesised that supplementation with the inulin prebiotic would beneficially shift the faecal microbiota, resulting in an increased relative abundance of Bifidobacterium, Faecalibacterium, Akkermansia and Lachnospira and a decreased relative abundance of Fusobacterium and Desulfovibrio, these changes were not observed in this study. Although those taxa were not altered, there was a decrease in Coprobacillus and there tended to be an increase in Eubacterium and Turicibacter in response to treatment with prebiotic. In a recent study by Garcia-Mazcorro et al. ${ }^{(22)}$, various breeds of dogs were supplemented with a prebiotic blend, containing inulin, making up approximately $0.5 \%$ of the diet on a DM basis. Similar to the present study, no significant treatments clustered in the unweighted or weighted principal coordinate analysis plots. The microbial changes we observed in this study were not altered in that study ${ }^{(22)}$. The bacteria belonging to the family Erysipelotrichaceae possess bile-salt hydrolase genes, which may modify BA via deconjugation ${ }^{(64)}$. An increase in BA in the colon may allow bile-metabolising bacteria such as those present in the Erysipelotrichaceae family to flourish. In this study, we show that the relative abundance of some Erysipelotrichaceae increased while others decreased, suggesting that its overall effect on the host is unknown. There is little information about the significance of Turicibacter in the canine gastrointestinal tract, although Turicibacter is reported to be lower in 
dogs with GI inflammation ${ }^{(65)}$. In contrast, another study reported that bacteria belonging to the family Turicibacteraceae were more abundant in dogs with active inflammatory colorectal polyps compared with healthy controls ${ }^{(66)}$. Although overweight, all dogs in the current study were otherwise healthy, so the relevance of this change in abundance in this study is unknown. Although substantial changes to the relative abundance of faecal microbial taxa were not observed, changes in gene expression/ functional capacity of the microbiome may be important and should be examined in future studies.

There were some important study limitations that probably contributed to the lack of significant results. After study completion, a retrospective power analysis for ANOVA was conducted using G*Power (Heinrich-Heine University). IAUC data for glucose, insulin and active GLP-1, as well as faecal total SCFA data, were used to determine effect size. Although we estimated that 9 dogs were needed per treatment to reach a power of $80 \%$, retrospective power analysis reveals that the predicted number of animals was too small, and a power of only $40 \%$ was achieved. Lack of significant outcomes with respect to the second-meal effect may be a result of underpowering. In addition, although a lower dose of the prebiotic was intentionally selected to represent a feasible inclusion level from a consumer (cost) prospective, it was insufficient to produce significant results.

In summary, this study suggests that low doses of prebiotic inulin are not sufficient to produce a significant second-meal effect in overweight dogs. As the study was underpowered, as analysed by a retrospective power analysis, future trials with increased animal or human participant numbers are warranted. The prebiotic did result in an increase in relative abundance of some members of the phylum Firmicutes and a decrease in the relative abundance of some Proteobacteria. In addition, supplementation with the prebiotic tended to increase the total concentration of excreted faecal BA, particularly secondary BA. These findings provide valuable information that may be used to design future studies investigating the ability of different prebiotics to produce a second-meal effect and the dosage required. Further studies in dogs and humans may provide new insights as to how prebiotic fibres can contribute to a secondmeal effect and elicit shifts in the gastrointestinal microbiome and BA pool.

\section{Acknowledgements}

The authors sincerely thank Kiley Algya, Toshiro Baba, Laura Bauer, Amanda Dainton, Katelyn Detweiler, Chelsea Iennarella, Daisy Ignatova, Jessica Kordas, Anne Lee, Ching-Yen Lin, Lexi Meverden, Juliana Nogueira, Thunyaporn Phungviwatnikul, Karine Santos and Zachary Traughber for their involvement in sample collections.

This study was supported by Beneo GmBH.

K. S. S. designed the experiment. C. A., T.-W. L. C. and S. D. performed the animal trials and laboratory analyses. C. A. performed the statistical analysis and wrote the manuscript.

F. N. and S. T. are employed by Beneo GmBH. The rest of the authors have no conflicts of interest to declare.

\section{Supplementary material}

For supplementary material/s referred to in this article, please visit https://doi.org/10.1017/S0007114518001952

\section{References}

1. Kaneko JJ, Mattheeuws D, Rottiers RP, et al. (1977) Glucose tolerance and insulin response in diabetes mellitus of dogs. J Small Anim Pract 18, 85-94.

2. Chikamune T, Katamoto H, Ohashi F, et al. (1995) Serum lipid and lipoprotein concentrations in obese dogs. J Vet Med Sci $\mathbf{5 7 ,} 595-598$.

3. Chandler M, Cunningham S, Lund EM, et al. (2017) Obesity and associated comorbidities in people and companion animals: a one health perspective. J Comp Pathol 156, 296-309.

4. Massimino SP, McBurney MI, Field CJ, et al. (1998) Fermentable dietary fiber increases GLP-1 secretion and improves glucose homeostasis despite increased intestinal glucose transport capacity in healthy dogs. J Nutr 128, 1786-1793.

5. Chandalia M, Garg A, Lutjohann D, et al. (2000) Beneficial effects of high dietary fiber intake in patients with type 2 diabetes mellitus. $N$ Engl J Med 342, 1392-1398.

6. Respondek F, Swanson KS, Belsito KR, et al. (2008) Shortchain fructooligosaccharides influence insulin sensitivity and gene expression of fat tissue in obese dogs. J Nutr 138, 17121718.

7. Weickert M, Möhlig M, Schöfl C, et al. (2006) Cereal fiber improves whole-body insulin. Diabetes Care 29, 773-780.

8. Reimer R \& McBurney M (1996) Dietary fiber modulates intestinal proglucagon messenger ribonucleic acid and postprandial secretion of glucagon-like peptide-l and insulin in rats. Endocrinology 137, 3948-3956.

9. Delzenne NM \& Cani PD (2005) A place for dietary fibre in the management of the metabolic syndrome. Curr Opin Clin Nutr Metab Care 8, 636-640.

10. Staub H (1921) Untersuchungen uber den Zuckerstoffwechsel des Munchen (Studies on the sugar metabolism of the munich). Z Klin Med 91, 44-48.

11. Traugott K (1922) Über das Verhalten des Blutzucherspiegels bei wiederholter und verschiedener Art enteraler Zuckerzufuhr und dessen Bedeutung für die Leberfunktion (On the behaviour of blood sugar levels with repeated and various types of enteral sugar intake and its importance for liver function). Klin Woch 1, 892-894.

12. Deng P, Beloshapka AN, Vester Boler BM, et al. (2013) Dietary fibre fermentability but not viscosity elicited the 'second-meal effect' in healthy adult dogs. Br J Nutr 110, 960-968.

13. Brighenti F, Benini L, Del Rio D, et al. (2006) Colonic fermentation of indigestible carbohydrates contributes to the second-meal effect. Am J Clin Nutr 83, 817-822.

14. Nilsson AC, Ostman EM, Holst JJ, et al. (2008) Including indigestible carbohydrates in the evening meal of healthy subjects improves glucose tolerance, lowers inflammatory markers, and increases satiety after a subsequent standardized breakfast. J Nutr 138, 732-739.

15. Priebe MG, Wang H, Weening D, et al. (2010) Factors related to colonic fermentation of nondigestible carbohydrates of a previous evening meal increase tissue glucose uptake and moderate glucose-associated inflammation. Am J Clin Nutr 91, 90-97.

16. de Carvalho CM, de Paula TP, Viana LV, et al. (2017) Plasma glucose and insulin responses after consumption of breakfasts with different sources of soluble fiber in type 2 diabetes 
patients : a randomized crossover clinical trial. Am J Clin Nutr 106, 1238-1245.

17. Ropert A, Cherbut C, Roze C, et al. (1996) Colonic fermentation and proximal gastric tone in humans. Gastroenterology 111, 289-296.

18. Robertson MD (2007) Metabolic cross talk between the colon and the periphery: implications for insulin sensitivity. Proc Nutr Soc 66, 351-361.

19. Anderson JW \& Bridges SR (1984) Short-chain fatty acid fermentation products of plant fiber affect glucose metabolism of isolated rat hepatocytes. Proc Soc Exp Biol Med 177, 372-376.

20. Middelbos IS, Boler BMV, Qu A, et al. (2010) Phylogenetic characterization of fecal microbial communities of dogs fed diets with or without supplemental dietary fiber using 454 pyrosequencing. PLOS ONE 5, e9768.

21. Beloshapka AN, Dowd SE, Suchodolski JS, et al. (2013) Fecal microbial communities of healthy adult dogs fed raw meat-based diets with or without inulin or yeast cell wall extracts as assessed by 454 pyrosequencing. FEMS Microbiol Ecol 84, 532-541.

22. Garcia-Mazcorro JF, Barcenas-Walls JR, Suchodolski JS, et al. (2017) Molecular assessment of the fecal microbiota in healthy cats and dogs before and during supplementation with fructooligosaccharides (FOS) and inulin using high-throughput 454-pyrosequencing. PeerJ 5, e3184.

23. Holscher HD, Bauer LL, Gourineni V, et al. (2015) Agave inulin supplementation affects the fecal microbiota of healthy adults participating in a randomized, double-blind, placebocontrolled, crossover trial. J Nutr 145, 2025-2032.

24. Holscher HD, Caporaso JG, Hooda S, et al. (2015) Fiber supplementation influences phylogenetic structure and functional capacity of the human intestinal microbiome: follow-up of a randomized controlled trial. Am J Clin Nutr 101, 55-64.

25. Dominianni C, Sinha R, Goedert JJ, et al. (2015) Sex, body mass index, and dietary fiber intake influence the human gut microbiome. PLOS ONE 10, e0124599.

26. Ridlon JM, Harris SC, Bhowmik S, et al. (2016) Consequences of bile salt biotransformations by intestinal bacteria. Gut Microbes 7, 22-39.

27. Joyce SA \& Gahan CGM (2016) Bile acid modifications at the microbe-host interface: potential for nutraceutical and pharmaceutical interventions in host health. Annu Rev Food Sci Technol 7, 313-333.

28. National Research Council (2006) Nutrient Requirements of Dogs and Cats. Washington, DC: National Research Council of the National Academies.

29. Barry KA, Hernot DC, Middelbos IS, et al. (2009) Low-level fructan supplementation of dogs enhances nutrient digestion and modifies stool metabolite concentrations, but does not alter fecal microbiota populations. J Anim Sci 87, 3244-3252.

30. Apanavicius CJ, Powell KL, Vester BM, et al. (2007) Fructan supplementation and infection affect food intake, fever, and epithelial sloughing from Salmonella challenge in weanling puppies. J Nutr 137, 1923-1930.

31. Association of Official Analytical Chemists (2006) Official Methods of Analysis. Arlington, VA: AOAC.

32. American Association of Cereal Chemists (1983) Approved Methods. St Paul, MN: AACC.

33. Budde EF (1952) The determination of fat in baked biscuit type of dog foods. J Assoc Off Agric Chem 35, 799-805.

34. Prosky L, Asp N-G, Schweizer TF, et al. (1992) Determination of soluble and insoluble dietary fiber in foods and food products: collaborative study. J AOAC 75, 360-367.

35. Knapp BK, Parsons CM, Swanson KS, et al. (2008) Physiological responses to novel carbohydrates as assessed using canine and avian models. J Agric Food Chem 56, 7999-8006.
36. Lubbs DC, Vester Boler BM, Ridge TK, et al. (2010) Dietary macronutrients and feeding frequency affect fasting and postprandial concentrations of hormones involved in appetite regulation in adult dogs. J Anim Sci 88, 3945-3953.

37. Erwin ES, Marco GJ \& Emery EM (1961) Volatile fatty acid analyses of blood and rumen fluid by gas chromatography. J Dairy Sci 44, 1768-1771.

38. Chaney AL \& Marbach EP (1962) Modified reagents for determination of urea and ammonia. Clin Chem 8, 130-132.

39. Flickinger EA, Schreijen EMWC, Patil AR, et al. (2003) Nutrient digestibilities, microbial populations, and protein catabolites as affected by fructan supplementation of dog diets. J Anim Sci 81, 2008-2018.

40. Kakiyama G, Muto A, Takei H, et al. (2014) A simple and accurate HPLC method for fecal bile acid profile in healthy and cirrhotic subjects: validation by GC-MS and LC-MS. J Lipid Res 55, 978-990.

41. McInnes P \& Cutting M (2010) Manual of Procedures for Human Microbiome Project - Core Microbiome Sampling Protocol A \#07-001, vol. 9. Bethesda, MD: National Institutes of Health.

42. Caporaso JG, Lauber CL, Walters WA, et al. (2012) Ultra-highthroughput microbial community analysis on the Illumina HiSeq and MiSeq platforms. ISME J 6, 1621-1624.

43. Malinen E, Rinttilä T, Kajander K, et al. (2005) Analysis of the fecal microbiota of irritable bowel syndrome patients and healthy controls with real-time PCR. Am J Gastroenterol 100, 373-382.

44. Rossi G, Pengo G, Caldin M, et al. (2014) Comparison of microbiological, histological, and immunomodulatory parameters in response to treatment with either combination therapy with prednisone and metronidazole or probiotic VSL \#3 strains in dogs with idiopathic inflammatory bowel disease. PLOS ONE 9, e94699.

45. Suchodolski JS, Markel ME, Garcia-Mazcorro JF, et al. (2012) The fecal microbiome in dogs with acute diarrhea and idiopathic inflammatory bowel disease. PLOS ONE 7, e51907.

46. Caporaso JG, Kuczynski J, Stombaugh J, et al. (2010) QIIME allows analysis of high-throughput community sequencing data intensity normalization improves color calling in SOLiD sequencing. Nat Publ Gr 7, 335-336.

47. Edgar RC (2010) Search and clustering orders of magnitude faster than BLAST. Bioinformatics 26, 2460-2461.

48. DeSantis TZ, Hugenholtz P, Larsen N, et al. (2006) Greengenes, a chimera-checked 16S rRNA gene database and workbench compatible with ARB. Appl Environ Microbiol 72, 5069-5072

49. Lozupone C \& Knight R (2005) UniFrac: a new phylogenetic method for comparing microbial communities. Appl Environ Microbiol 71, 8228-8235.

50. Deng P, Jones JC \& Swanson KS (2014) Effects of dietary macronutrient composition on the fasted plasma metabolome of healthy adult cats. Metabolomics 10, 638-650.

51. Parks DH, Tyson GW, Hugenholtz P, et al. (2014) STAMP: Statistical analysis of taxonomic and functional profiles. Bioinformatics 30, 3123-3124.

52. Mollard RC, Wong CL, Luhovyy BL, et al. (2014) Second-meal effects of pulses on blood glucose and subjective appetite following a standardized meal 2 h later. Appl Physiol Nutr Metab 39, 849-851.

53. Clark C, Gardiner J, McBurney M, et al. (2006) Effects of breakfast meal composition on second meal metabolic responses in adults with type 2 diabetes mellitus. Eur J Clin Nutr 60, 1122-1129.

54. Roediger WEW (1982) Utilization of nutrients by isolated epithelial cells of the rat colon. Gastroenterology 83, 424-429. 
55. Liong M-TT (2007) Probiotics: a critical review of their potential role as antihypertensives, immune modulators, hypocholesterolemics, and perimenopausal treatments. Nutr Rev 65, 316-328.

56. Topping DL \& Clifton PM (2001) Short-chain fatty acids and human colonic function: roles of resistant starch and nonstarch polysaccharides. Physiol Rev 81, 1031-1064.

57. Cummings J \& Macfarlane G (1991) The control and consequences of bacterial fermenation in the human colon. J Appl Bacteriol, 443-459.

58. Macfarlane GT \& Allison C (1986) Utilisation of protein by human gut bacteria. FEMS Microbiol Ecol 38, 19-24.

59. Ridlon JM, Kang D-J \& Hylemon PB (2006) Bile salt biotransformations by human intestinal bacteria. J Lipid Res $\mathbf{4 7}$, 241-259.

60. Suchodolski JS (2016) Diagnosis and interpretation of intestinal dysbiosis in dogs and cats. Vet J 215, 30-37.

61. Washizu T, Ikenaga H, Washizu M, et al. (1990) Bile acid composition of dog and cat gall-bladder bile. Jpn J Vet Sci 52, 423-425.
62. Honneffer J, Guard B, Steiner JM, et al. (2015) Untargeted metabolomics reveals disruption within bile acid, cholesterol, and tryptophan metabolic pathways in dogs with idiopathic inflammatory bowel disease. Gastroenterology 148, S-715.

63. Rémésy C, Levrat M, Gamet L, et al. (1993) Cecal fermentations in rats fed oligosaccharides (inulin) are modulated by dietary calcium level. Am J Physiol 264 , G855-G862.

64. Labbe A, Ganopolsky JG, Martoni CJ, et al. (2014) Bacterial bile metabolising gene abundance in Crohn's, ulcerative colitis and type 2 diabetes metagenomes. PLOS ONE 9, e115175.

65. AlShawaqfeh M, Wajid B, Minamoto Y, et al. (2017) A dysbiosis index to assess microbial changes in fecal samples of dogs with chronic inflammatory enteropathy. FEMS Microbiol Ecol 93, fix136.

66. Igarashi H, Ohno K, Horigome A, et al. (2016) Fecal dysbiosis in miniature dachshunds with inflammatory colorectal polyps. Res Vet Sci 105, 41-46. 